\title{
Single port robotic radical cystectomy with intracorporeal urinary diversion: a case series and review
}

\author{
Michael Zhang ${ }^{1}$, Devon Thomas ${ }^{2}$, George Salama ${ }^{3}$, Mutahar Ahmed $^{4}$ \\ ${ }^{1}$ Department of Urology, New York Medical College, Valhalla, NY, USA; ${ }^{2}$ St. Georges University, Grenada, West Indies; ${ }^{3}$ Department of \\ Urology, Rutgers New Jersey Medical School, Newark, NJ, USA; ${ }^{4}$ The Center for Bladder Cancer at Hackensack University Medical Center, \\ Hackensack, NJ, USA \\ Contributions: (I) Conception and design: M Ahmed; (II) Administrative support: None; (III) Provision of study material or patients: M Ahmed; (IV) \\ Collection and assembly of data: D Thomas, G Salama; (V) Data analysis and interpretation: M Zhang, M Ahmed; (VI) Manuscript writing: All \\ authors; (VII) Final approval of manuscript: All authors. \\ Correspondence to: Mutahar Ahmed, MD, FACS. Director of The Center for Bladder Cancer at Hackensack University Medical Center, Hackensack, \\ NJ, USA. Email: drahmedgu@gmail.com.
}

\begin{abstract}
The da Vinci single port surgical system (SP) is the newest iteration of robotic technology, which combines the camera and all instruments into a single port. Robotic assisted radical cystectomy (RARC) with intracorporeal urinary diversion has classically been a difficult operation. Here we describe our technique for RARC with the SP and review our initial outcomes and data in the literature. Four patients at our institution underwent single port robotic assisted radical cystectomy with intracorporeal ileal conduit using the da Vinci SP surgical system. Operative steps were performed as described. Perioperative outcomes were reviewed. All patients successfully underwent the procedure without intraoperative complications. The average operative time was 270 minutes with an EBL of $250 \mathrm{cc}$. The average nodal harvest was 12.5 . The average length of stay was 5.5 days. There was one 30-day Clavien Grade II complication and no additional 90-day complications. With our initial experience with the da Vinci surgical system, radical cystectomy with intracorporeal urinary diversion can be performed safely and quickly with the right technique. Our outcomes are similar to other initially published data. Further study is necessary to determine the additional benefits and clinical outcomes.
\end{abstract}

Keywords: Single port; robotic cystectomy; intracorporeal urinary diversion

Submitted Sep 30, 2019. Accepted for publication Jan 14, 2020.

doi: $10.21037 /$ tau.2020.01.19

View this article at: http://dx.doi.org/10.21037/tau.2020.01.19

\section{Introduction}

Radical cystectomy is the gold standard treatment for muscle invasive and refractory noninvasive bladder cancer, but it remains a morbid operation with high potential for complications and readmissions (1). In the last 10 years there has been a steady rise in the use of robotic assisted radical cystectomy (RARC) in an effort to enhance recovery times and decrease patient morbidity. SEER data shows an increase in RARC incidence from $0.7 \%$ of all cystectomies in 2002 to $18.5 \%$ of all cystectomies in 2012 (2). In that time, there has been a steady improvement in technique, from RARC with extracorporeal urinary diversion as described by Menon et al. (3) to RARC with completely intracorporeal urinary diversion. Recent data from the RAZOR trial, which compared outcomes from RARC to open RC, showed equivalent oncologic outcomes between the two arms at two years of follow up (4). However, despite the intuitive benefits of the more minimally invasive approach, RARC did not seem to reduce the overall complication rate and had a minimally improved length of stay.

Last year, the da Vinci Single Port Surgical System was approved by the FDA for urologic procedures. It represents the next step in minimally invasive surgery and combines the robotic camera as well as three instrument slots into a 
single $25 \mathrm{~mm}$ multichannel port. By further minimizing the invasiveness of RARC, the SP has potential to further reduce morbidity associated with this procedure. Our institution has a robust robotics experience, with over ten years of experience in RARC and five years of experience with intracorporeal urinary diversion. In this article, we describe our initial experience with performing SP RARC, including our stepwise approach, perioperative outcomes and review current publications.

\section{Methods}

Between April-August 2019, our institution has performed four single port robotic cystectomies with completely intracorporeal ileal conduit. All cases were performed by the same surgeon using the da Vinci Single Port Surgical System. Perioperative outcomes were reviewed and analyzed. IRB approval was obtained as part of our prospective cystectomy database. Here we describe our surgical technique as well as surgical considerations (see Video 1, detailing complete procedure).

\section{Patient positioning}

Male patients undergoing RC are placed in supine position while female patients are placed in dorsal lithotomy position to allow for access to the vagina. Arms are tucked to the side and pressure points are padded. All patients are placed in the Trendelenburg position during the case to allow for easier management of bowel.

\section{Trocar placement}

An initial $3 \mathrm{~cm}$ incision is made $3-4 \mathrm{~cm}$ above the umbilicus. Entry into the abdomen is obtained through the Hassan technique. The fascial incision is shifted $1 \mathrm{~cm}$ inferiorly compared to the skin incision to allow the robotic trocar to come in at an angle. A mini GelPOINT advanced access platform is used to house the $25-\mathrm{mm}$ robotic trocar. Finally, a 12-mm SurgiQuest AirSeal port is placed at the marked stoma site (see Figure 1).

\section{General considerations}

The optimal working length of the SP robot instruments is $10-25 \mathrm{~cm}$. Using the GelPOINT allows the robotic trocar to be burped in and out of the body. This adjustment increases the working range of the instruments and is imperative in order to reach deep into the pelvis for the most distal points of dissection and to retract backwards to allow for bowel work in the proximal abdomen.

We found that our optimum instrument position is the robotic camera at the 120 -clock position, a monopolar scissor at the 6 o-clock position, a bipolar grasper at the 9 o-clock position, and the Cadiere forceps at the 3 o-clock position. We find that this positioning allows for minimal instrument swapping for the entirely of the case and minimizes instrument collisions.

\section{Right ureteral dissection}

We begin our right-sided ureteral dissection by opening the peritoneum just lateral to the medial umbilical ligament. This incision is carried caudally to expose the retroperitoneum on the right side. The ureter is found as it crosses the iliac vessels, then is freed down to the right ureterovesical junction (UVJ). Great care is taken to ensure a meticulous dissection of the ureter by sparing the peri-ureteral tissue. We then free the lateral side wall of the bladder and follow the medial umbilical ligament posteriorly. This dissection will help establish the borders for the lymph node dissection. In doing this, the lateral pedicle of the bladder is also exposed and freed. The ureter is then clipped and transected, and a segment of ureter is removed and sent to pathology for frozen section. In females, the infundibulopelvic ligament is divided, as the ureter runs underneath this structure.

\section{Right pelvic lymph node dissection}

Pre-operative decision making is required to delineate the proximal extent of dissection. Our standard template reaches up to the level of the common iliac vessels, but extended dissections can be used for more aggressive disease (5). We begin by carefully dissecting the lymphatic tissue at the level of the common iliac vessels on the right side using the "split and roll" technique. As this is the most proximal extent of dissection, it may be necessary to burp the robotic trocar almost out of the body to allow for the minimum $10 \mathrm{~cm}$ working distance. The dissection is then taken distally. The common iliac vessels are followed until its bifurcation. The internal iliac (hypogastric) nodes are the medial border of the pelvic lymph node dissection and are carefully removed. The external iliac nodes are taken down distally to the level of the circumflex vessels. The external iliac vessels are mobilized medially to expose the pelvic 

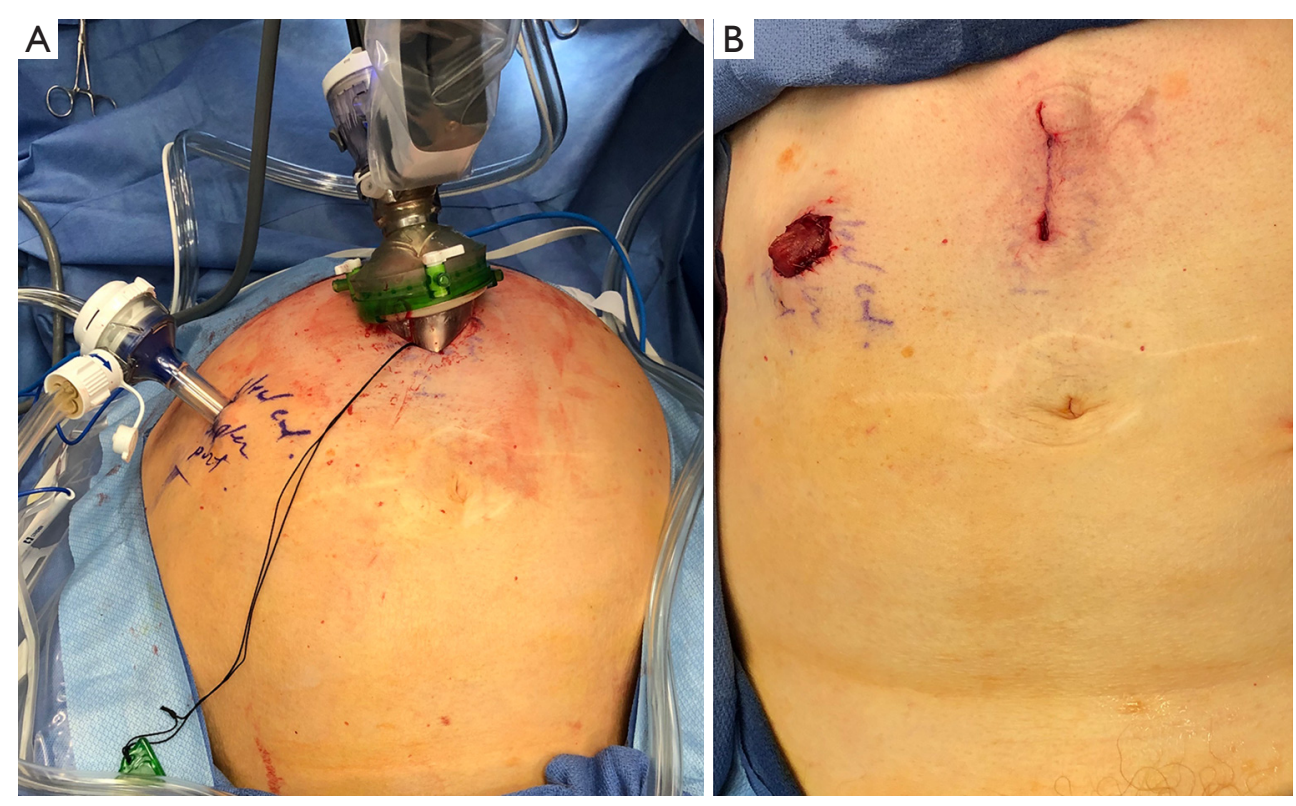

Figure 1 Instrument positioning. (A) Port arrangement with AirSeal port at eventual stoma site; (B) appearance after procedure is complete.

side wall along with the proximal aspect of the obturator nerve. This mobilization will simplify the removal of the obturator and hypogastric nodes by allowing these packets to be pulled from the underside of the external iliac vein. The Space of Marseille should be cleared of nodal tissue. Utilizing the Cadiere helps to retract the external iliac artery and vein medially to expose the space and remove nodes from this complex area.

\section{Left ureteral mobilization/pelvic lymph node dissection}

Overall mobilization of left ureter is similar to the right side. The sigmoid colon is first mobilized medially in order to better identify the left ureter, which runs more medially compared to the right ureter. The distal ureter is then freed and transected after being clipped. The left side is taken more proximally compared to right side to allow for passage of the ureter under the sigmoid mesentery. The left pelvic lymph node dissection is also performed in a similar fashion to the right side. If a super-extended template is employed the IMA will be visualized on this side. The left ureter is then transferred under the sigmoid mesentery, anterior to the aorta, to the right side.

\section{Pre-rectal space and posterior bladder dissection}

In males, the peritoneum between bladder and rectum is carefully incised in a similar fashion to the "posterior approach" to the robotic radical prostatectomy. This is done while providing upwards traction on the bladder away from the rectum. Visualization of the vas deferens and seminal vesicles helps to delineate the proper plane. Denonvilliers fascia is opened, and the dissection is carried out as distally as possible towards the apex of the prostate.

In females, sponge stick or metal EEA is used to identify the vaginal. The peritoneum posterior to the bladder is opened and the vagina is opened onto the sponge stick at the level of the anterior fornix. The posterior vagina is carefully dissected away from rectum as lateral as possible. Once the bladder and anterior vaginal wall are completely separated from posterior vagina, the posterior cuff is closed on itself in a "clam-shell" or longitudinal fashion.

A vagina-sparing approach can be employed in select female patients. By identifying and developing the avascular plane between anterior vagina and bladder, the urethra/ sphincter can be spared. This is necessary if planning to perform a neobladder.

\section{Division of bladder pedicles}

The bladder pedicles are easily defined after thorough and appropriate posterior/lateral dissection. The endopelvic fascia is opened and the superior and inferior bladder pedicles are cauterized using bipolar energy before being 


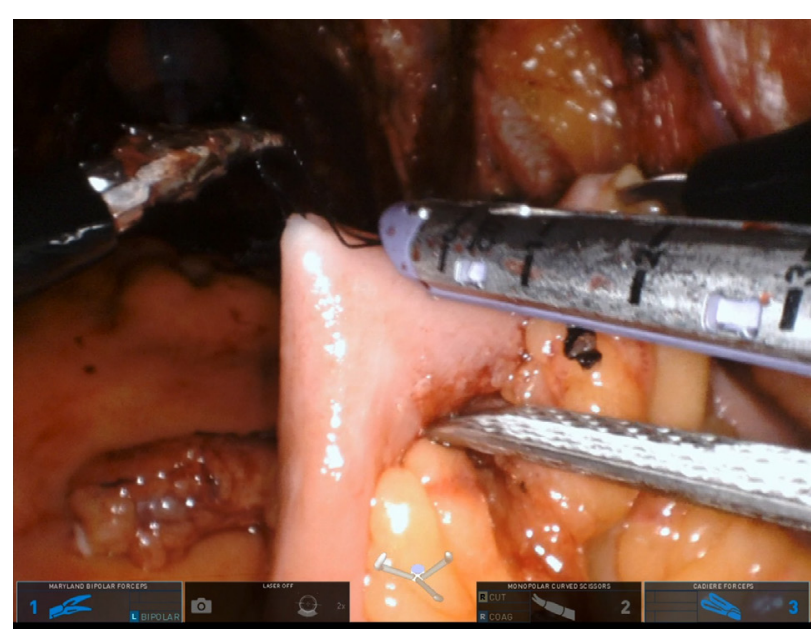

Figure 2 Use of stay sutures to arrange bowel to allow for division with stapler.

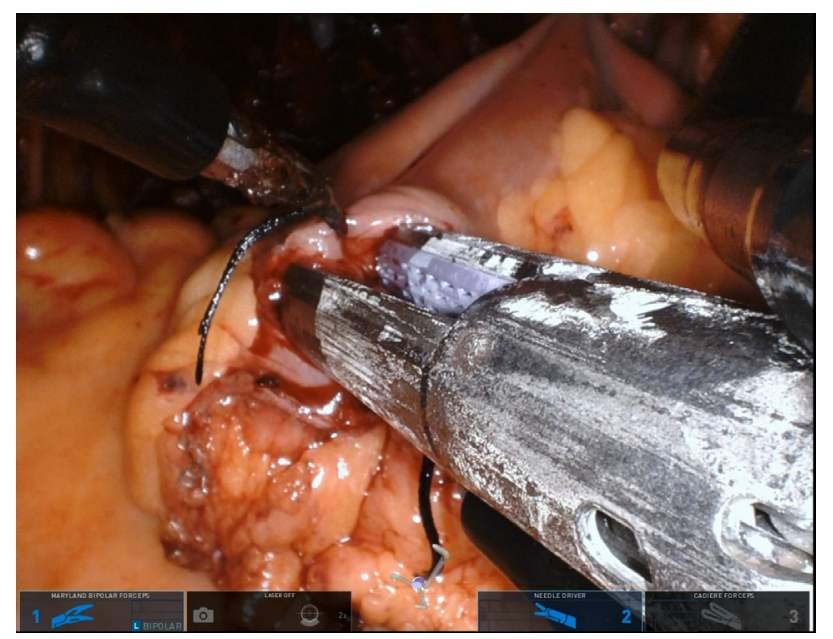

Figure 3 Holding the bowel deep in the pelvis and angling the enterotomies towards the assistant port to allow for bowel anastomosis.

divided. This is done segmentally in order to ensure hemostasis. If performing nerve sparing, the nerves are mobilized off the pedicle in a retrograde fashion using hemo-lock clips instead of thermal energy.

\section{Anterior bladder dissection}

The median umbilical ligaments are divided and the space of retzius is entered in a similar fashion to a robotic prostatectomy. Any remaining endopelvic fascia or puboprostatic ligaments are divided, then the dorsal venous complex is ligated using bipolar energy and sharp cutting. The rectourethralis muscle is then carefully dissected away from urethra. A generous urethral stump is necessary if planning for a neobladder. Once the specimen is freed, it is placed in a specimen bag. In females, this is removed through the vagina prior to vaginal closure.

\section{Intracorporeal ileal conduit creation}

The terminal ileum is identified by locating the appendix or cecum. A stay suture is used to mark the distal portion of the ileal conduit at $20-25 \mathrm{~cm}$ proximal to the terminal ileum. A second stay suture is placed $12-16 \mathrm{~cm}$ proximal to first suture, marking the future proximal conduit segment. The bowel is then divided using a Covidien EndoGIA $60 \mathrm{~mm}$ stapler (Figure 2). The mesentery at each end is divided using bipolar energy to allow for later mobilization up to the skin. Care must be taken to avoid jeopardizing bowel perfusion. In a multiport RARC, ICG can be used at this point to confirm good bowel perfusion. Unfortunately, firefly capability is still under development with the single port system and is currently unavailable.

Once the conduit segment is mobilized, attention is turned to bowel re-anastomosis. Prior to this step, it is important to place multiple stay sutures to line up the bowel in order to provide stability and mobilization. The last stay suture must be past the anticipated staple line to allow for proper tensioning of the bowel during stapling. Small enterotomies are made at the anti-mesenteric portion of each bowel loop. The bowel segments are then placed as deep in the pelvis as possible and angled up towards the $12 \mathrm{~mm}$ assistant port. One robotic arm is used to hold the most distal stay suture and another is used to hold the most proximal stay suture while the assistant fires the handheld stapler. One staple load is used to establish continuity between the two segments and another load is used for end sealing (Figure 3). The mesentery of the re-aligned anastomosed bowel segment is over sewed to prevent internal hernias.

The ureters are then brought over to the conduit segment and adequate mobilization is confirmed by maintaining the ureters off tension. We prefer a Wallace combined ureteroileal anastomosis for our intracorporeal urinary diversions. Both ureters are spatulated and the posterior wall of each segment is brought together using 4-0 monocryl. A small enterotomy on butt end of the conduit is 
Table 1 Prospectively gathered patient characteristics and postoperative outcomes

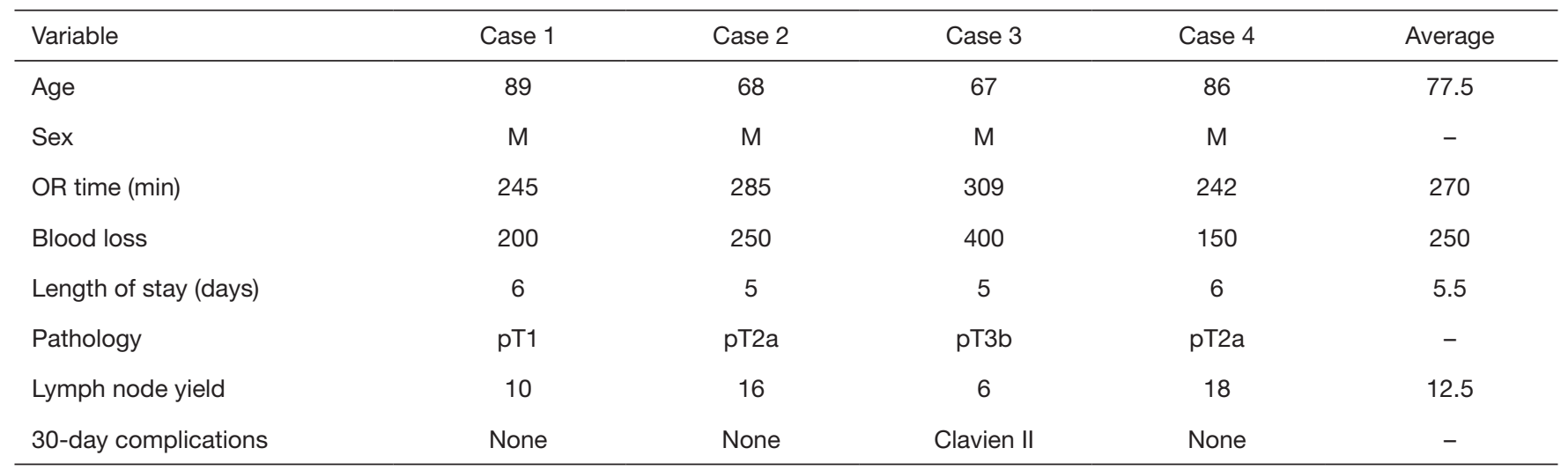

OR, operating room.

made. The posterior edge of the anastomosis is run before placing two JJ or urinary diversion stents in the ureters. The anastomosis is then complete by running the anterior edge. The assistant port is then removed and a laparoscopic needle driver is placed into the abdomen and the stay suture of the distal conduit is secured onto the needle driver. We then place a Jackson-Pratt drain through a separate stab incision to minimize the leakage of serous fluid around the drain. The robot is then undocked and the stoma is matured.

\section{Intracorporeal neobladder creation}

Although we have not yet performed this urinary diversion using the SP, the approach remains the same as the multiport approach. The terminal ileum is identified and a stay suture is placed $20 \mathrm{~cm}$ proximal to mark the distal end of the neobladder. A second stay suture is placed $54-60 \mathrm{~cm}$ proximal to the first and used to mark the proximal end of the neobladder. The mesentery is divided at each location in a similar fashion to an ileal conduit creation. The Covidien EndoGIA stapler is $60 \mathrm{~mm}$ stapler is used to isolate the marked segment of bowel. Bowel re-anastomosis is established using the technique as described above. The urethroenteric anastomosis is started by making an enterotomy in a bowel segment that easily reaches urethra, usually the midsection of the isolated bowel segment. The anastomosis is performed with 3-0 V-lock suture. The rest of the bowel segment is then opened along the antimesenteric border in an "upside down U" configuration. The posterior wall of the neobladder is closed with 3-0 monocryl, followed by the anterior wall. Ureteroenteric anastomosis is performed using either the Wallace or Bricker technique and internal double-J stent are placed. A foley is then placed and the neobladder is irrigated to check for leak.

\section{Results}

Overall, four single port robotic radical cystectomies with intracorporeal ileal conduit were performed over a five month period. All cases were successfully completed without conversion to open or conversion to multiport. The average operative time was 270 minutes and the average length of stay was 5.5 days (see Table 1). There was one 30-day complication-a Clavien grade II (blood transfusion). There were no additional 90-day complications.

\section{Discussion}

The goal of all minimally invasive surgery is to perform an oncologically sound operation while minimalizing morbidity to the patient. Recent studies have shown equivalent oncologic outcomes between RARC and open $\mathrm{RC}$, but did not show a reduction in overall complication rate and had minimal improvement in length of stay (3). Ahmed et al. compared the outcomes of intracorporeal vs. extracorporeal urinary diversion and found no difference in the 90-day complication rate, but did find a lower 90 -day readmission rate with the intracorporeal approach (OR 0.68) (6).

The single port surgical system offers improved cosmesis over open and standard robotic approaches, but has potential to offer decreased pain and reduce other complications as well. 
Due to the recentness of the da Vinci SP robot, there is limited data on outcomes associated with SP RARC. Kaouk et al. published a four patient series on their initial experience with the operation. They showed an average operative time of 454 minutes with only one Clavien grade 1 complication. However, they had one patient with conversion to open urinary diversion (7).

In addition, Garisto et al. published a cadaveric case of a transperineal single port cystoprostatectomy with intracorporeal urinary diversion. Whether this leads to functional or clinical outcomes has yet to be seen (8).

Our data shows that SP RARC with intracorporeal urinary diversion is safe and feasible for experienced high volume robotic surgeons. Further study is necessary to determine if the approach will lead to reduced morbidity or improved perioperative outcomes.

\section{Acknowledgments}

Funding: None.

\section{Footnote}

Provenance and Peer Review: This article was commissioned by the Guest Editor (Ashok K. Hemal) for the series "Robotic-assisted Urologic Surgery" published in Translational Andrology and Urology. The article was sent for external peer review organized by the Guest Editor and the editorial office.

Conflicts of Interest: All authors have completed the ICMJE uniform disclosure form (available at http://dx.doi. org/10.21037/tau.2020.01.19). The series "Robotic-assisted Urologic Surgery" was commissioned by the editorial office without any funding or sponsorship. The authors have no other conflicts of interest to declare.

Ethical Statement: The authors are accountable for all aspects of the work in ensuring that questions related to the accuracy or integrity of any part of the work are appropriately investigated and resolved.

Open Access Statement: This is an Open Access article distributed in accordance with the Creative Commons Attribution-NonCommercial-NoDerivs 4.0 International License (CC BY-NC-ND 4.0), which permits the noncommercial replication and distribution of the article with the strict proviso that no changes or edits are made and the original work is properly cited (including links to both the formal publication through the relevant DOI and the license). See: https://creativecommons.org/licenses/by-nc-nd/4.0/.

\section{References}

1. Bishoff JT, Kavoussi LR. Atlas of laparoscopic and robotic urologic surgery. Philadelphia, PA: Elsevier, 2017.

2. Hu JC, Chughtai B, O'Malley P, et al. Perioperative Outcomes, Health Care Costs, and Survival After Robotic-assisted Versus Open Radical Cystectomy: A National Comparative Effectiveness Study. Eur Urol 2016;70:195-202.

3. Menon M, Hemal AK, Tewari A, et al. Nerve-sparing robot-assisted radical cystoprostatectomy and urinary diversion. BJU Int 2003;92:232-6.

4. Parekh DJ, Reis IM, Castle EP, et al. Robot-assisted radical cystectomy versus open radical cystectomy in patients with bladder cancer (RAZOR): an open-label, randomised, phase 3, non-inferiority trial. Lancet 2018;391:2525-36.

5. Hwang EC, Sathianathen NJ, Imamura M, et al. Extended versus standard lymph node dissection for urothelial carcinoma of the bladder in patients undergoing radical cystectomy. Cochrane Database Syst Rev 2019;5:CD013336.

6. Ahmed K, Khan SA, Hayn MH, et al. Analysis of intracorporeal compared with extracorporeal urinary diversion after robot-assisted radical cystectomy: results from the International Robotic Cystectomy Consortium. Eur Urol 2014;65:340-7.

7. Kaouk J, Garisto J, Eltemamy M, et al. Single-port Robotic Intracorporeal Ileal Conduit Urinary Diversion During Radical Cystectomy Using the SP Surgical System: Step-by-step Technique. Urology 2019;130:196-200.

8. Garisto J, Bertolo R, Chan E, et al. Single-Port TransPerineal Approach to Cystoprostatectomy with Intracorporeal Ileal Conduit Urinary Diversion and Lymph-Nodes Dissection using a Purpose-Built Robotic System: Surgical Steps in a Preclinical Model. Int Braz J Urol 2019;45:854-5.

Cite this article as: Zhang $M$, Thomas D, Salama G, Ahmed M. Single port robotic radical cystectomy with intracorporeal urinary diversion: a case series and review. Transl Androl Urol 2020;9(2):925-930. doi: 10.21037/tau.2020.01.19 